\title{
Effect of Reduced Grapheme Oxide in Enhancing the Photocatalytic Activity of $\beta-\mathrm{NaYF}_{4}: \mathrm{Ho}^{3+} @ \mathrm{TiO}_{2}$
}

\author{
Wanyu Han*, Tianhui Wu, Shuang Zhu \\ Power China Huadong Engineering Corporation Limited, Hangzhou, China \\ Email address: \\ han_wy@ecidi.com (Wanyu Han),wu_th@ecidi.com (Tianhui Wu), zhu_s@ecidi.com (Shuang Zhu) \\ ${ }^{*}$ Corresponding author
}

To cite this article:

Wanyu Han, Tianhui Wu, Shuang Zhu. Effect of Reduced Grapheme Oxide in Enhancing the Photocatalytic Activity of $\beta-\mathrm{NaYF}_{4}: \mathrm{Ho}^{3+} @ \mathrm{TiO}_{2}$. American Journal of Water Science and Engineering. Vol. 5, No. 2, 2019, pp. 88-95. doi: 10.11648/j.ajwse.20190502.15

Received: May 9, 2019; Accepted: June 11, 2019; Published: July 4, 2019

\begin{abstract}
NaYF}_{4}: \mathrm{Ho}^{3+} @ \mathrm{TiO}_{2}$-reduced graphene oxide (NYFH@TO-rGO) ternary composites photocatalysts were prepared via a three-step method and used for cleanup of Rhodamine $\mathrm{B}(\mathrm{RhB})$ aqueous solution under visible light irradiation. X-ray diffraction (XRD), scanning electron microscopy (SEM), energy-dispersive X-ray spectroscopy (EDS), fluorescence spectrometries, ultraviolet-visible diffuse reflectance spectroscopy (UV-Vis DRS), electron spin resonance (ESR), and photoelectrochemical properties were used to characterize the photocatalyst. The results revealed that rGO as an excellent platform and successfully to load NYFHo@TO core-shell microcrystals. In this photocatalyst, the loading of UC microcrystals is expected to emit UV $(290 \mathrm{~nm})$ light after absorbing Vis $(450 \mathrm{~nm})$ light of the solar spectrum and the optical response of the rGO is enhanced from UV to Vis. so as to realize the visible light-driven photocatalysis of $\mathrm{TiO}_{2}$. It was found that add to $\mathrm{rGO}$ can efficient charge separation, extended light absorption range (red-shifted to $402.6 \mathrm{~nm}$ ), enhanced adsorption performance, and improve photocatalytic activity. This novel tenary photocatalyst can reach decomposition rate of $\mathrm{RhB}$ as high as $87 \%$ after $10 \mathrm{~h}$ of irradiation by visible-light Xe lamp. Compared with the blank experiment, the efficiency was significantly improved. It is of great significance to design an effective solar light-driven photocatalysis in promoting environmental protection.
\end{abstract}

Keywords: Wastewater Treatment, Upconversion, Visible Light Photocatalysis

\section{Introduction}

Since Fujishima and Honda [1] discovered the phenomenon of photocatalytic splitting of water on a $\mathrm{TiO}_{2}$ electrode under ultraviolet (UV) light [2], $\mathrm{TiO}_{2}$ has been widely studied in areas ranging from photocatalysis [3-5]. However, because of the wide band gap of $\mathrm{TiO}_{2}\left(\mathrm{E}_{\mathrm{g}}=3.0-3.2 \mathrm{eV}\right)$, only a wavelength of less than $387 \mathrm{~nm}$ UV light, which only makes up ca. $5 \%$ of solar energy could produce effective photocatalytic activity [6]. Furthermore, the fast recombination of charge carriers significantly reduces the catalytic activity in practical applications. As a result, large scale practical application of $\mathrm{TiO}_{2}$-based photocatalysts still needs further innovational work [7].

Recently, there have been reports of the combination of upconversion luminescence (UC) agents with the semiconductors, which allowed the use of a visible or NIR light source for catalysis [8-10]. In order to efficiently utilize the valuable upconversion energy transferred from the excited upconversion core [11]. The core-shell structure has potential, which can protect the upconversion core from surface quenching and increase the energy transfer efficiency to the photocatalyst shell. However, recently studies have focused on the doped $\mathrm{Yb}^{3+}$ ion in the UC matrix material and only excitation at $980 \mathrm{~nm}$ NIR [12-15]. Its UC efficiency is limited with NIR radiation source excitation, mainly because of its extremely weak absorption in the NIR wavelength range. We had studied about $\beta-\mathrm{NaYF}_{4}: \mathrm{Ho}^{3+} @ \mathrm{TiO}_{2}$ core-shell microcrystals and the successful use of Vis as a photocatalyst [16]. However, there are still some problems about $\mathrm{UC} @ \mathrm{TiO}_{2}$ core-shell structures, and most serious problem is the fast recombination of photogenerated electron-hole pairs. So, other methods are still needed found to improvement these problems. 
Graphene (GR) and reduced graphene oxide (rGO) have emerged as attractive candidates for constructing graphenebased materials because it has several valuable characteristics [17-19]. First, GR materials exhibit high electron mobility and extended $\pi$-electron conjugation. In addition, GR has a high specific surface area and the unique flexible sheet-like structure of the GR component. Recently, peoples pay much attention to $\mathrm{TiO}_{2}$-grapheme (T@GR) which has shown a significant improvement of the photoelectrochemical catalytic ability [20-22]. Research indications GR not only has a high theoretical specific surface area, can increase the adsorption surface of the catalyst, but also can improve the adsorption performance of $\mathrm{TiO}_{2}$ by chemical adsorption. The T@GR composite has the characteristics of high light transmittance and zero bandgap of GR, and due to the excellent electrical conductivity for storing and shuttling electrons (flexible sheet-like structure), which can inhibiting the recombination of photogenerated electron-hole pairs. Moreover, the formation of $\mathrm{Ti}-\mathrm{O}-\mathrm{C}$ bonds can expand the light absorption to longer wavelengths [23]. Despite all the advantages, these T@GR composites still suffer from a key factor that limits the photocatalytic activity under solar irradiation, i.e. the low-usage of natural sunlight.

Herein, we combined the above-mentioned strategies together and demonstrated for the first time a NYFH@TO core-shell structure supported on rGO as a new Vis-driven photocatalyst. Benefiting from the high specific surface area and the flexible sheet-like structure, rGO emerged as an excellent platform on which to load NYFHo@TO core-shell microcrystals to form $\beta-\mathrm{NaYF}_{4}: \mathrm{Ho}^{3+} @ \mathrm{TiO}_{2}$-reduced graphene oxide (NYFH@TO-rGO) ternary composites. In this photocatalyst, the loading of UC microcrystals is expected to emit UV light after absorbing Vis light of the solar spectrum and the optical response of the NYFH@TOrGO microcrystals is enhanced from UV to Vis. At the same time, rGO can inhibit the recombination of photogenerated electron-hole pairs and enhance the adsorption capacity of the photocatalyst. Finally, the photodegradation of rhodamine $\mathrm{B}(\mathrm{RhB})$ has been investigated.

\section{Experimental Section}

\subsection{Synthesis of NYFH@TO-rGO Ternary Composites}

The NYFH@TO core-shell microcrystals synthesis method reference group of preliminary work [16]. UV-irradiation of the NYFH@TO-graphene oxide (NYFH@TO-GO) samples was performed using an Oriel $500 \mathrm{~W}$ xenon arc lamp. Firstly, $2 \mathrm{mg} / \mathrm{mL}$ GO $(2.5 \mathrm{~mL})$ was ultrasonicated in a $100 \mathrm{~mL}$ of anhydrous ethanol solution to disperse it well; after that,0.1 g of NYFH@TO was added to the above GO solution and vigorous stirring $1 \mathrm{~h}$. A water filter, which was used to cutoff IR, assisted in maintaining the temperature of the ethanol sample $(300 \mathrm{~K})$ during irradiation with minimum variation in the experimental results. The suspensions were kept agitated during UV irradiation through bubbling of the solution with nitrogen gas.
The agitation of the samples ensured uniform irradiation of the NYFH@TO-GO suspension during the reduction process. The precipitate was centrifuged and washed with deionized water two times and dried in the vacuum freeze drier at $-60^{\circ} \mathrm{C}$ for $24 \mathrm{~h}$, resulting in NYFH@TO-rGO ternary composites.

\subsection{Photocatalytic Activity Measurement}

The photocatalytic activity of the NYFH@TO-rGO ternary composite was measured via comparing the concentration of rhodamine $\mathrm{B}(\mathrm{RhB})$ after irradiation to the original concentration of $\mathrm{RhB}$, by measuring optical absorption using a Hitachi U-3010 UV-Vis spectrophotometer (Hitachi Corp., Tokyo, Japan) at $553 \mathrm{~nm}$. Typically, $50 \mathrm{mg}$ of photocatalyst was suspended in $250 \mathrm{~mL}$ $\mathrm{RhB}$ aqueous solution $(5 \mathrm{mg} / \mathrm{L})$ by sonication. Prior to irradiation, the suspension was stirred in the dark for $0.5 \mathrm{~h}$ to establish the adsorption-desorption equilibrium. Then the solution was exposed to the irradiation of a $500 \mathrm{~W}$ Xenon arc lamp with a UV cutoff filter $(\lambda>420 \mathrm{~nm})$. Every 2 hour, 8 $\mathrm{mL}$ of the transparent, aqueous solution was collected and then centrifuged $(10,000 \mathrm{r} / \mathrm{min})$ prior to analysis with the Hitachi U-3010 UV-Vis spectrophotometer.

\subsection{Characterization}

The crystal structures of all prepared samples were characterized by X-ray diffraction (XRD) using a Rigaku D/Max2500pc diffractometer with $\mathrm{Cu} K \alpha$ radiation. Scanning electron microscopy (SEM) images were acquired with a Zeiss AURIGA FE microscope $(\mathrm{EHT}=5 \mathrm{kV}$, WD = $8.8 \mathrm{~nm}$; Zeiss, Oberkochen, Germany). Fourier transform infrared spectroscopy (FT-IR, IRPrestige-21, Shimadzu, Japan) analysis of the composite was performed using FT-IR spectrophotometer $(\mathrm{KBr}$ as the reference sample). UV-Vis diffuse-reflectance spectroscopy (UV-Vis DRS) was performed with the Hitachi U-3010 UV-Vis spectrophotometer. The sample for electron spin resonance (ESR) measurement was prepared by mixing NYFH@TOrGO samples in a $50 \mathrm{mM}$ DMPO solution tank (aqueous dispersion for DMPO- $\cdot \mathrm{OH}$ and methanol dispersion for DMPO- $\mathrm{O}_{2}^{-}$). Photoelectrochemical properties were evaluated using a CHI Electrochemical Workstation (CHI 760E, Shanghai Chenhua, China). All the photoelectrochemical measurements were performed under Vis light of a $300 \mathrm{~W}$ Xe lamp coupled with 420 nmcutoff filters. All experiments were performed at room temperature.

\section{Results and Discussion}

\subsection{Structural and Morphology Characterization}

The phase structures of the samples were characterized by XRD measurements, and the XRD patterns of the NYFH, NYFH@TO, and NYFH@TO-rGO are shown in Figure 1. The results show that all the diffraction peaks of the NYFH could be assigned to hexagonal phase $\beta-\mathrm{NaYF}_{4}$ (JCPDS. 
16-0334), which is the best UC luminescence of the matrix materials [24]. The diffraction peaks of anatase $\mathrm{TiO}_{2}$ (JCPDS no. 21-1272) were detected in the samples of NYFH@TO and NYFH@TO-rGO indicates that anatase $\mathrm{TiO}_{2}$ and hexagonal phase $\mathrm{NaYF}_{4}$ coexist. However, no diffraction peak typical of $\mathrm{rGO}$ appeared in the XRD patterns of the samples. We attributed this result to the fact that the amount of rGO was below the limit of detection (LOD) for XRD. The relative intensities of some of the diffraction peaks differed between the standard and the prepared products, which were indicative of differences in the degree of crystallization and morphology among the obtained products.

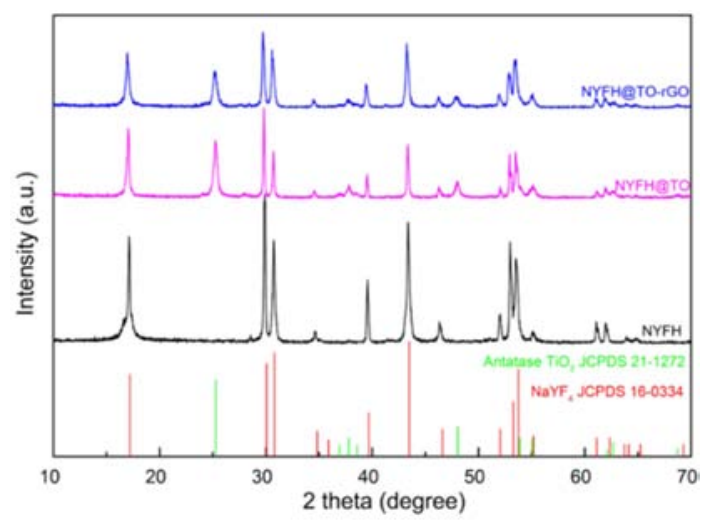

Figure 1. The XRD patterns of NYFH, NYFH@TO, and NYFH@TO-rGO.
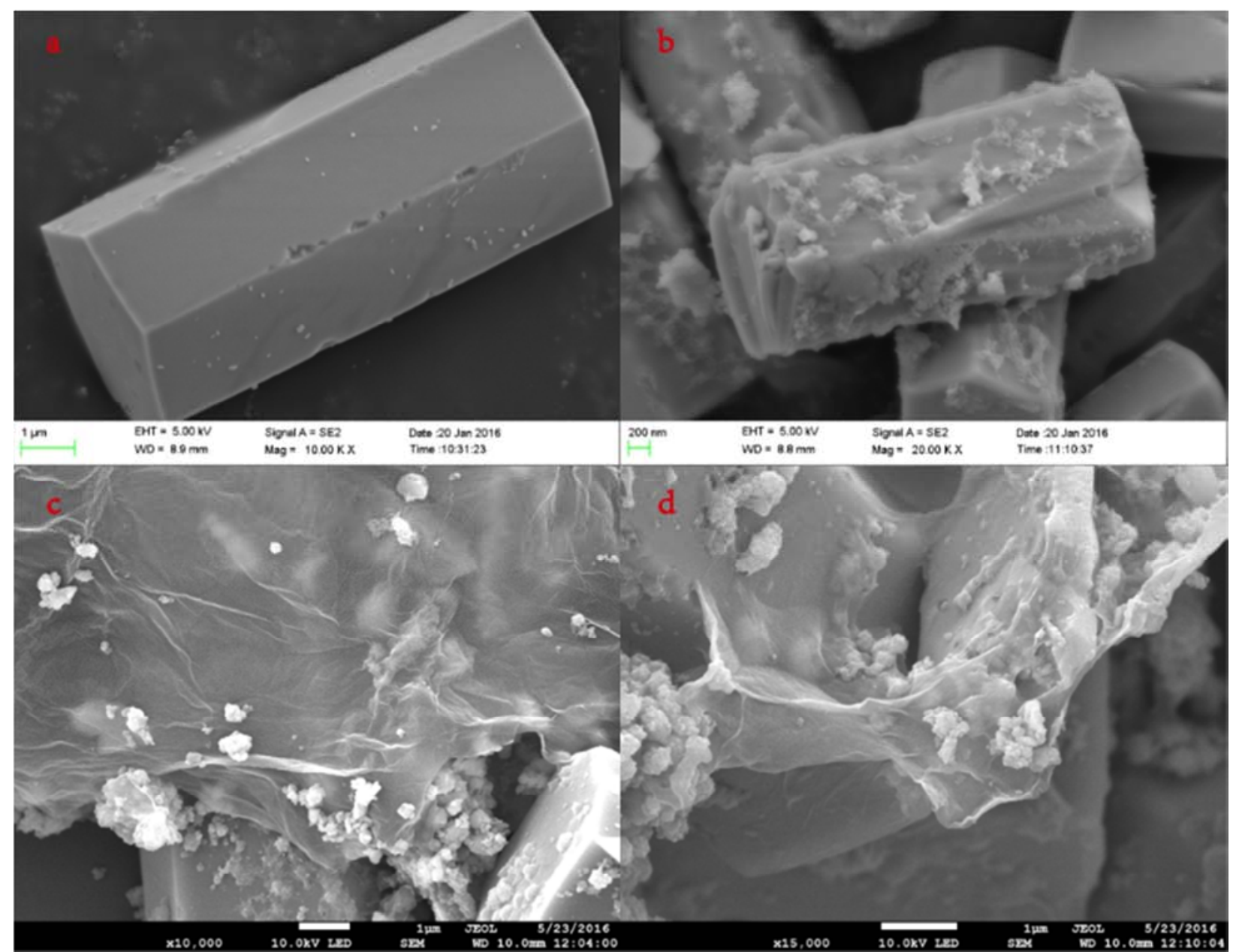

Figure 2.SEM images of (a) NYFH, (b) NYFH@TO, and (c-d) NYFH@TO-rGO

The morphologies of the prepared samples were examined by SEM. Figure 2 shows SEM images of different samples. As shown in Figure 2a, pure NYFH particles had uniform size and shape distributions and formed a series of hexagonal prisms-like structures and the average microcrystal length is roughly $8 \mathrm{um}$, with a diameter of about $2.4 \mathrm{um}$. All the images show that the surfaces of the microcrystals are very smooth. After coating, the surfaces become coarser (Figure $2 \mathrm{~b}$ ), showing that the microcrystals are successfully coated with a $\mathrm{TiO}_{2}$ layer. It is also found that some agglomeration of
$\mathrm{TiO}_{2}$ is attached to NYFH surface. Figure 2c shows that some NYFH@TO was loaded on the rGO sheets and few $\mathrm{TiO}_{2}$ nanoparticles were dispersed on the surface of the rGO sheets. As shown in the magnified SEM images (Figure 2d), the rGO sheets were inserted between the NYFH@TO, and wrap up a part of NYFH@TO. This structure creates a good interface between NYFH@TO core-shell microcrystals, and rGO to facilitate efficient charge transport within the composite, which leads to efficient separation of photogenerated carriers in the coupled rGO ternary composites. 

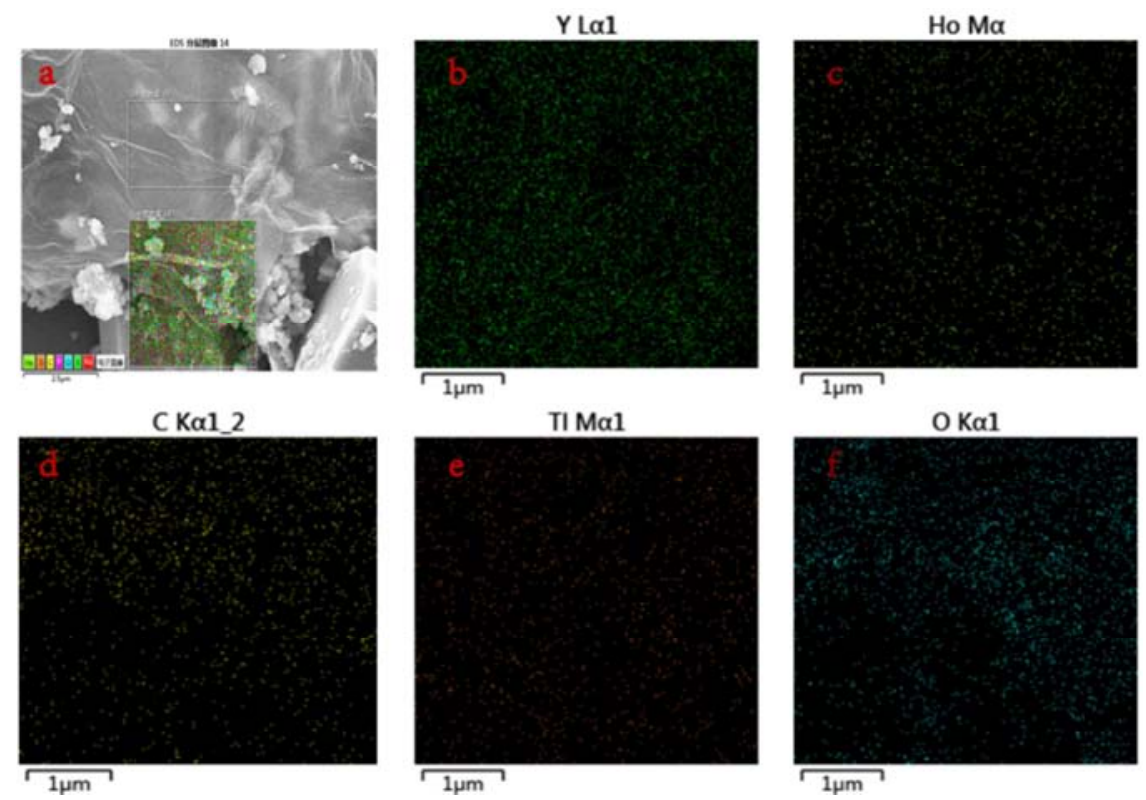

Figure 3. (a) SEM images of NYFH@TO-rGO microcrystals, (b-f) corresponding SEM elemental distribution mappings of Y, Ho, C, Na, Ti, and O.

SEM coupled with energy dispersive X-ray spectroscopy (EDX) was used to further verify the elemental composition of the as-synthesized ternary composites. Results are shown in Figure 3. Figure 3b-f shows the corresponding elemental mappings for the elements $\mathrm{Y}, \mathrm{Ho}, \mathrm{C}, \mathrm{Ti}$, and $\mathrm{O}$, respectively. We found some interesting results from these images. For examples, we can easily find that $\mathrm{Y}, \mathrm{Ho}, \mathrm{Ti}$, and $\mathrm{O}$ are homogeneously distributed throughout the space. However, C elemental the upper part is obviously more than the lower part, from the Figure $3 \mathrm{a}$ we find that the upper part of the region was covered with $\mathrm{rGO}$, also the $\mathrm{rGO}$ sheets below cover with NYFH@TO. All the results further confirm that $\mathrm{TiO}_{2}$ and hexagonal-phase $\mathrm{NaYF}_{4}$ core-shell microcrystals coexist in the rGO platform and formation ternary composites. These results are consistent with the results of XRD.

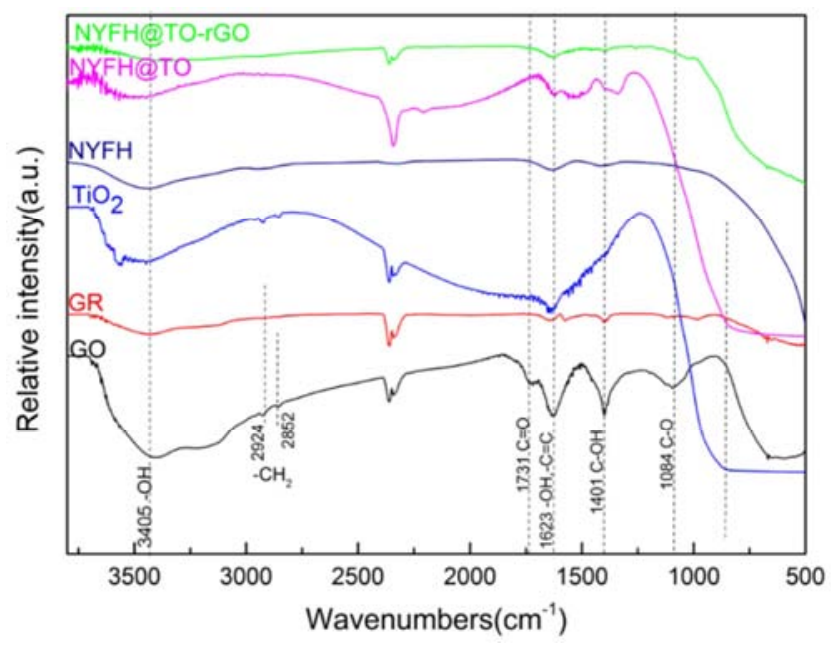

Figure 4. FTIR spectra of GO,GR, TiO $, N Y F H, N Y F H @ T O$, and NYFH@TO-rGO.
In order to ensure the efficient transfer of GR and characterize the carbon species, Further insights into the reduction of GO was obtained by FTIR. Figure 4 shows the FTIR spectra of GO, GR, TiO 2, NYFH, NYFH@TO, and NYFH@TO-rGO composites, respectively. GO shows a strong absorption band at $3405 \mathrm{~cm}^{-1}$ due to the $\mathrm{O}-\mathrm{H}$ stretching vibration. It also the characteristic peaks appearing at $1731,1623,1401,1224$ and $1084 \mathrm{~cm}^{-1}$ can be assigned to carboxyl or carbonyl $\mathrm{C}=\mathrm{O}$ stretching, $\mathrm{H}-\mathrm{O}-\mathrm{H}$ bending band of the adsorbed $\mathrm{H}_{2} \mathrm{O}$ molecules, carboxyl $\mathrm{O}-\mathrm{H}$ stretching, phenolic $\mathrm{C}-\mathrm{OH}$ stretching and alkoxy $\mathrm{C}-\mathrm{O}$ stretching, respectively[25, 26]. Compared with the GO, various oxygen-containing groups $\left(800-1900 \mathrm{~cm}^{-1}\right)$ in NYFH@TO-rGO and GR has a significant decrease and even absent, suggesting that the UV-Assisted photocatalytic treatment is an effective method for the reduction of GO to rGO. In the case of the GR- $\mathrm{TiO}_{2}$ composite, the typical absorption peaks of GO decrease dramatically in intensity or even disappear as compared with those of the pure GO, indicating the reduction of GO. The broad absorption below $800 \mathrm{~cm}^{-1}$ is ascribed to the vibration of Ti-O-Ti bonds and Ti-O-C in $\mathrm{TiO}_{2}$ [27], corresponding to that in spectrum of $\mathrm{TiO}_{2}$ and NYFH@TO. Meanwhile, the formation of Ti-O-C bonds can expand the light absorption to longer wavelengths [23]. All the results show that a ternary composite catalyst for the $\mathrm{rGO}$ as a platform was successfully prepared.

\subsection{The Effect of Rgo Enhancement Photocatalyst Activity}

According to the original idea, the introduction of $\mathrm{rGO}$ as a photocatalyst platform in the NYFH@HO system to efficient charge separation, extended light absorption range, enhanced adsorption performance, and improve photocatalytic activity. In order to ensure NYFH@HO-rGO ternary composite have the above characteristics, a series of characterization were employed. 

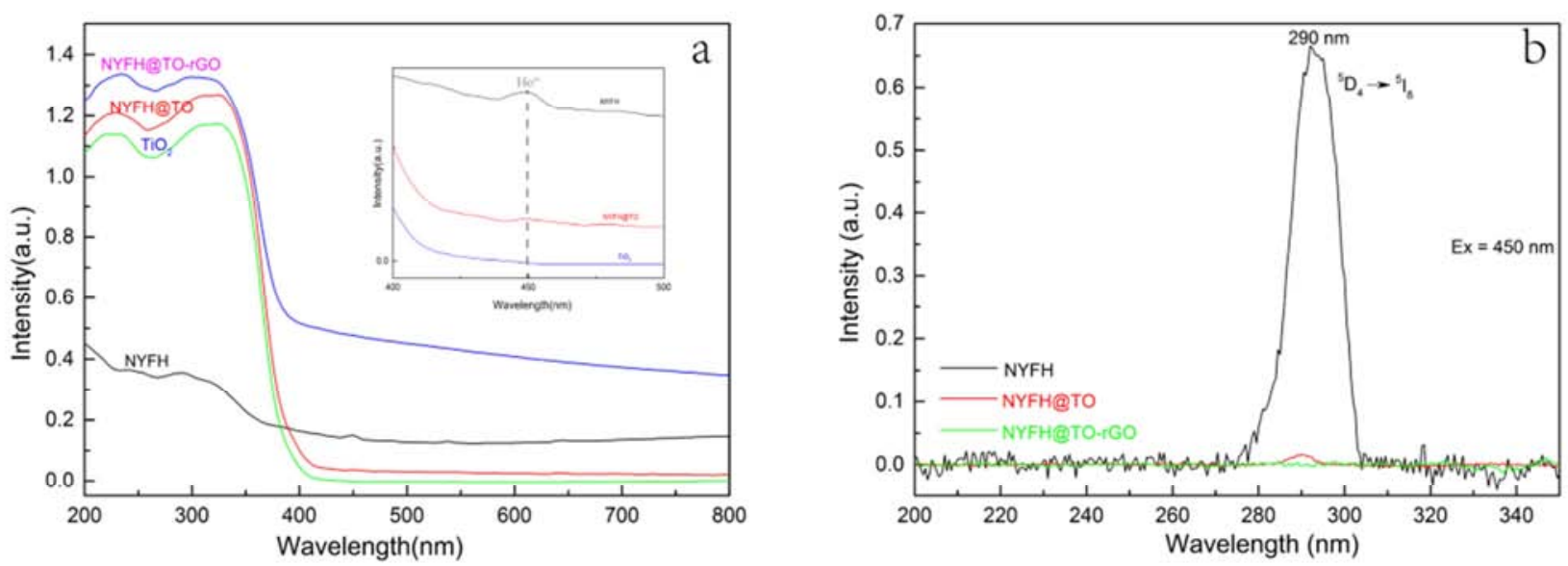

Figure 5. (a) The UV-Vis absorbance spectra of the TiO2, NYFH, NYFH@TO and NYFH @,TO-rGO; inset: the enlarge spectra ranging from 400 nm to 500 nm. (b) Photoluminescence (PL) spectra of samples under (b) $450 \mathrm{~nm}$ excitations.

We investigated the optimal absorption wavelength of the synthesized materials through UV-Vis absorption spectroscopic analysis. Figure 5a shows representative spectra of $\mathrm{TiO}_{2}$, NYFH, NYFH@TO and NYFH@TO-rGO. Notably, a light absorption edge before $400 \mathrm{~nm}$ which is overlapped with that of $\mathrm{TiO}_{2}$, corresponding to the band absorption of $\mathrm{TiO}_{2}(\leq 3.2 \mathrm{eV})$, is observed for the spectrum of NYFH@TO and NYFH@TO-rGO. Meanwhile, the NYFH@TO-rGO showed significant enhancement of light absorption at a wavelength of $400-800 \mathrm{~nm}$, which could be mainly attributed to absorption of visible light by the rGO itself. Also shown in the spectrum of $\beta-\mathrm{NaYF}_{4}: \mathrm{Ho}^{3+}$, corresponds to the absorption of $\mathrm{Ho}^{3+}$, which has absorption cross sections at about $450 \mathrm{~nm}$. Accordingly, considering the wavelength of the $450 \mathrm{~nm}$ were selected as the excitation wavelength. The bandgap of the $\mathrm{TiO}_{2}$, NYFH@TO, and NYFH@TO-rGO can be calculated by using Tauc's formula. The bandgaps of $\mathrm{TiO}_{2}, \mathrm{NYFH} @ \mathrm{TO}$, and NYFH@TO-rGO are calculated to be 3.22, 2.21, and 3.08

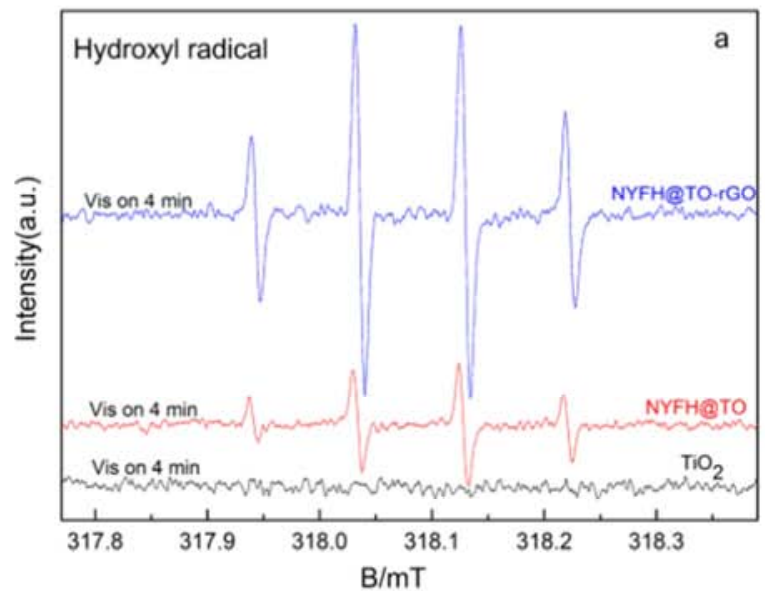

$\mathrm{eV}$, respectively. Notably, the absorption edge for the NYFH@TO-rGO composite was red-shifted compared to those of pure $\mathrm{TiO}_{2}$ and NYFH@TO, given that the absorption edges were measured to be at $385.09 \mathrm{~nm}$ for pure $\mathrm{TiO}_{2}, 386.29$ nm for NYFH@TO, and 402.60 nm for NYFH@TO-rGO.

UV UC emission by the $\mathrm{Ho}^{3+}$ ions was clearly observed. The UV emission band around $290 \mathrm{~nm}$ originated from the ${ }^{5} \mathrm{D}_{4} \rightarrow{ }^{5} \mathrm{I}_{8}$ transition of $\mathrm{Ho}^{3+}$ under $450 \mathrm{~nm}$ excitation, as shown in Figure 5b. After coating NYFH with $\mathrm{TiO}_{2}$, notable spectral differences are observed (Figure 5b). The intense emission peak at $290 \mathrm{~nm}$ almost disappears in comparison with the UC fluorescence spectra of NYFH microcrystals, indicating that the UC UV emission can be strongly quenched by being absorbed by $\mathrm{TiO}_{2}$. Furthermore, the UV emission was completely quenched in the case of NYFH@TO-rGO, indicating the incorporation of rGO further improve the energy transfer between NYFH and $\mathrm{TiO}_{2}$.

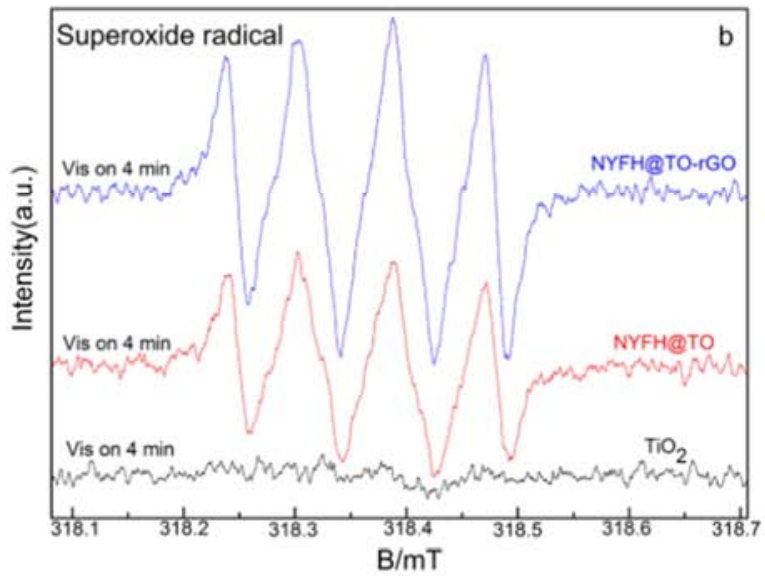

Figure 6. ESR spectral features of the DMPO-OH (a) and $\mathrm{DMPO}-\mathrm{O}_{2}^{-}$(b) spin adducts in the system under irradiation of full-spectrum Xe lamp with different photocatalystsTiO2,NYFH@TO, and NYFH@TO-rGO, respectively.

Moreover, the ESR method, widely used in photogenerated charge transfer processes, also revealed the key role of the UC materials in the photocatalytic degradation by the ESR spectra of the $\mathrm{OH}$ and $\cdot \mathrm{O}_{2}^{-}$radical $[28,29]$. The ESR spectral features of the DMPO-·OH and DMPO-- $\mathrm{O}_{2}^{-}$spin adducts in the system with different photocatalysts pure $\mathrm{TiO}_{2}$, NYFH@TO, 
and NYFH@TO-rGO are shown in Figure 6, respectively. After the same irradiation time, line $\mathrm{TiO}_{2}$ had no signal, while lines NYFH@TO and NYFH@TO-rGO displayed signals; which indicated that the $\cdot \mathrm{OH}$ and $\cdot \mathrm{O}_{2}{ }^{-}$radical was surely formed under the present conditions. This revealed that UC can convert Vis into UV to realize the photocatalysis of broadband $\mathrm{TiO}_{2}$. It can also be seen that the intensities of the signals increased in the presence of the UC materials, indicating that the concentrations of free radicals increased. The major reason for this phenomenon was that the $\mathrm{TiO}_{2}$ shell can efficiently absorb UV light (290 nm) emitted from the UC cores upon irradiation by a high pressure mercury lamp (500 W) with a UV cutoff filter $(\lambda>420 \mathrm{~nm})$. The presence of rGO can enhance UC luminescence to form stronger UV emissions; therefore, the intensity of line NYFH@TO-rGO $\cdot \mathrm{OH}$ and $\cdot \mathrm{O}_{2}{ }^{-}$ is 2.9 and 1.6 fold that of line NYFH@TO. This phenomenon was consistent with the result of the photocatalytic degradation, and it also revealed the key role of the UC materials in the photocatalytic degradation with NYFH@TO-rGO under non-UV excitation.

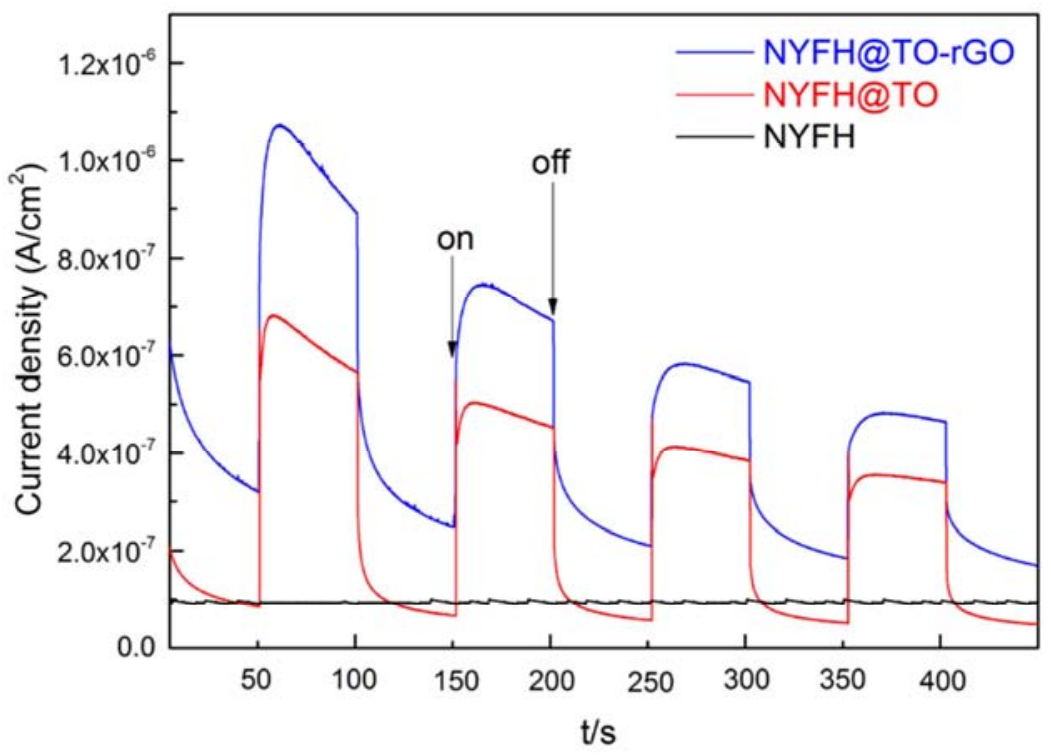

Figure 7. Transient photocurrent density of the as-prepared samples.

Photoelectrochemical measurements were performed to investigate the excitation, separation, transfer, and recombination of photoinduced charge carriers [30]. As shown in Figure 7, the photocurrent responses for NYFH@TO-rGO composite electrodes are prompt, steady and reproducible during repeated on/off cycles of the Vis irradiation. In contrast, no photocurrent response occurs for bare NYFH, and lower photocurrent density is found in the case of NYFH@TO. The enhancement of the photocurrent for the NYFH@TO-rGO electrode indicates a higher photoinduced charge separation efficiency and charge transfer rate in composite. The rGO serves as an acceptor and a transporter for the electrons generated from $\mathrm{TiO}_{2}$ shell after excited by upconversion energy from the NYFH core, thus inhibiting the recombination of photo-generated electron-hole pairs effectively.
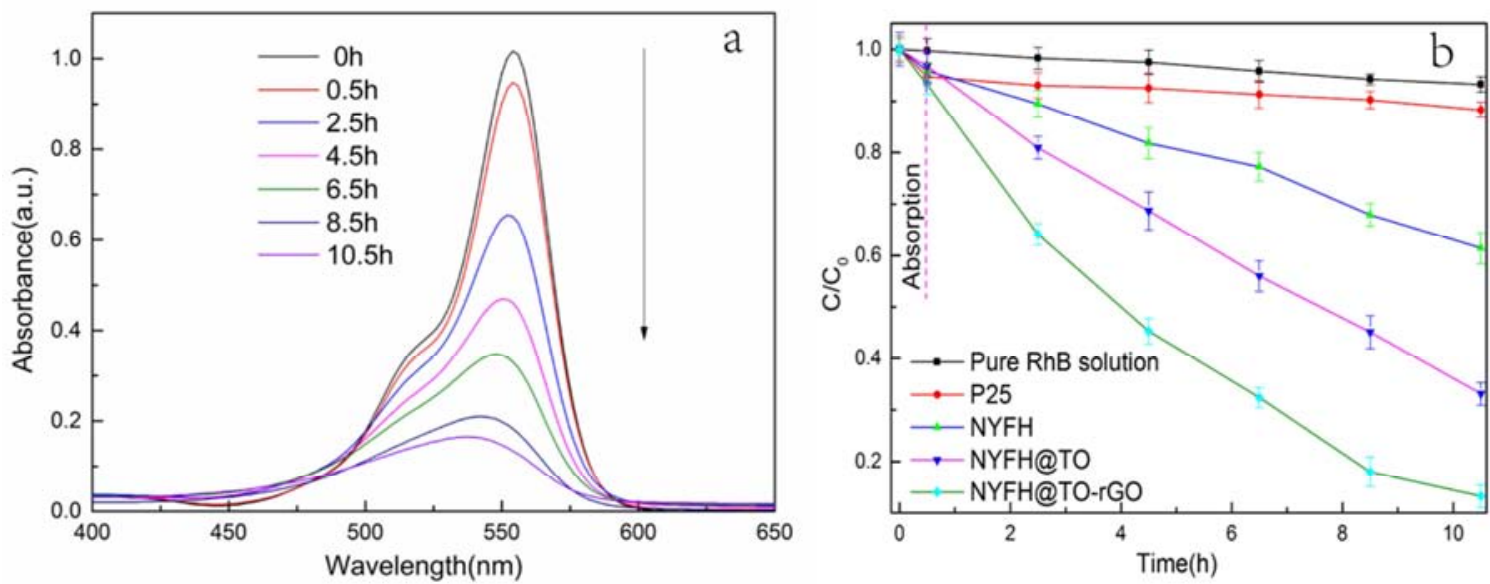

Figure 8. (a) Time-dependent UV-Vis absorption spectroscopy of a RhB solution degraded by NYFH@TO-rGO ternary composite. (b) Time-dependent plot of the photodegradation of an RhB solution by different catalysts upon irradiation by a Xe lamp (500 W) with a UV cutoff filter ( $\lambda>420 \mathrm{~nm})$. 
Figure 8a shows the absorbance spectra of RhB catalyzed by the NYFH@TO-rGO under Xe lamp irradiation as a function of the irradiation time. The absorption intensity of $\mathrm{RhB}$ at $554 \mathrm{~nm}$ decreases gradually with the increase in irradiation time, indicating the degradation of $\mathrm{RhB}$. As shown in Figure $8 \mathrm{~b}$, without catalyst, only about $7 \%$ of the $\mathrm{RhB}$ was decomposed under the Xe lamp irradiation $10 \mathrm{~h}$, while the degradation ratio of $\mathrm{RhB}$ reached $\sim 87 \%$ after $10 \mathrm{~h}$ irradiation in the presence of NYFH@TO-rGO ternary composite. It may be, on one hand, based on rGO strong adsorption performance. Surface adsorption of the dye is the key step for photocatalytic reaction and plays important roles in determining photocatalytic degradation activity [31]. On the other hand, extended light absorption range can let NYFH@TO-rGO make use of more light. With NYFH and NYFH@TO about 38 and 67\% of the RhB was decomposed after $10 \mathrm{~h}$ irradiation, respectively, which is far below the NYFH@TO-rGO. It is very clear that rGO as NYFH@TO core-shell structure catalysis platform is beneficial to photocatalytic activity according to the reaction results.

\section{Conclusions}

In this work, we have demonstrated a new strategy by integrating the Vis-to-UV UC property of NYFH with the excellent electrical properties of graphene to enhance the photocatalytic efficiency of $\mathrm{TiO}_{2}$. NYFH@TO-rGO ternary composites photocatalysts were prepared via a three-step method. The enhanced photocatalytic activity is associated with the large extended photoresponsive range, great adsorptivity of dyes and high electron-hole separation efficiency due to the synergetic interactions among $\mathrm{TiO}_{2}$, graphene and UC material. This work is anticipated to promote practical applications of photocatalysts under solar irradiation in addressing various environmental issues.

\section{References}

[1] Honda, A. et al. Electrochemical Photolysis of water at a semiconductor electrode. Nature. 238, 37-38 (1972).

[2] Chen XB, Liu L, et al. Properties of Disorder-Engineered Black Titanium Dioxide Nanoparticles through Hydrogenation. Scientific Reports, 3, 1510-1522 (2013).

[3] Li B, Zhang T. Biodegradation and adsorption of antibiotics in the activated sludge process. Environmental Science \& Technology, 44 (9): 3468-3473 (2010).

[4] Akpan UG, Hameed BH Parameters affecting the photocatalytic degradation of dyes using $\mathrm{TiO}_{2}$-based photocatalysts: a review. J Hazard Mater 170:520-529 (2009).

[5] Ambrus Z, Balázs N, Alapi T Synthesis, structure and photocatalytic properties of $\mathrm{Fe}$ (III)-doped $\mathrm{TiO}_{2}$ prepared from TiCl3. Appl Catal B 81:27-37 (2008).

[6] Tang, Y. et al. NIR-Responsive Photocatalytic Activity and Mechanism of $\mathrm{NaYF}_{4}: \quad \mathrm{Yb}, \quad \mathrm{Tm} @ \mathrm{TiO}_{2}$ CoreS-hell Nanoparticles. ACS Catal. 3, 405-412 (2013).
[7] Dorman JA, Weickert J, Reindl JB, et.al. Control of Recombination Pathways in $\mathrm{TiO}_{2}$ Nanowire Hybrid Solar Cells Using Sn4+ Dopants. J Phys Chem C 118:16672 (2014).

[8] Zhang, Y. \& Hong, Z. Synthesis of lanthanide-doped $\mathrm{NaYF}_{4} @ \mathrm{TiO}_{2}$ core-shell composites with highly crystalline and tunable $\mathrm{TiO}_{2}$ shells under mild conditions and their upconversion-based photocatalysis. Nanoscale. 5, 8930-8933 (2013).

[9] Guo, Xingyuan, et al. Near-infrared photocatalysis of $\beta-\mathrm{NaYF}_{4}: \mathrm{Yb}^{3+}, \mathrm{Tm}^{3+} @ \mathrm{ZnO}$ composites. Phys Chem Chem Phys 15:14681 (2013).

[10] Ma Y. M., Liu H. L., Mao M., Meng J., Yang L. B., Liu J. H. 'Surface-Enhanced Raman Spectroscopy on Liquid Interfacial Nanoparticle Arrays for Multiplex Detecting Drugs in Urine. Anal. Chem. 88: 8145-51, (2016).

[11] Guo, X. et al. Preparation and upconversion luminescence of $\beta-\mathrm{NaYF}_{4}: \mathrm{Yb}^{3+}, \mathrm{Tm}^{3+} / \mathrm{ZnO}$ nanoparticles. J. Nano. Nanotechnol. 14, 3726-3730 (2014).

[12] Guo, X. et al. Enhanced near-infrared photocatalysis of $\mathrm{NaYF}_{4}$ : $\mathrm{Yb}, \mathrm{Tm} / \mathrm{CdS} / \mathrm{TiO}_{2}$ composites. Dalton Trans. 43, 1048-1054 (2014).

[13] Chuanhao Li, Feng Wang, Jian Zhu, Jimmy C. Yu. $\mathrm{NaYF}_{4}: \mathrm{Yb}$, $\mathrm{Tm} / \mathrm{CdS}$ composite as a novel near-infrared-driven photocatalyst Applied Catalysis B: Environmental.100, 433-439 (2010).

[14] Wanjun Wang, Yecheng Li, Zhiwen Kang, et al. A NIR-driven photocatalyst based on $\alpha-\mathrm{NaYF}_{4}: \mathrm{Yb}, \mathrm{Tm} @ \mathrm{TiO}_{2}$ core-shell structure supported on reduced graphene oxide. Applied Catalysis B: Environmental.182: 184-192 (2016).

[15] Wu Tianhui, Long Jun, Xuan Xu. Synthesis and Photocatalytic Activity of Hexagonal Phase $\mathrm{NaYF}_{4}: \mathrm{Ho}^{3+} @ \mathrm{TiO}_{2}$ Core-ShellMicrocrystal. CrystEngComm, 18: $6471-6482$ (2016).

[16] Pengyu Dong, Yuhua Wang. Ag3PO4/reduced graphite oxide sheets nanocomposites with highly enhanced visible light photocatalytic activity and stability. Applied Catalysis B: Environmental.132: 45-53 (2013).

[17] Huanxin Zhao, Shuo Chen. Integration of microfiltration and visible-light-driven photocatalysis on g-C3N4 nanosheet/reduced graphene oxide membrane for enhanced water treatment. Applied Catalysis B: Environmental.194: 134-140 (2016).

[18] Chrysoula P. Athanasekou. Prototype composite membranes of partially reduced graphene oxide/ $\mathrm{TiO}_{2}$ for photocatalytic ultrafiltration water treatment under visible light. Applied Catalysis B: Environmental.159: 361-372(2014).

[19] Zhang, Y. \& Hong, Z. Synthesis of lanthanide-doped $\mathrm{NaYF}_{4} @ \mathrm{TiO}_{2}$ core-shell composites with highly crystalline and tunable $\mathrm{TiO}_{2}$ shells under mild conditions and their upconversion-based photocatalysis. Nanoscale. 5: 8930-8933 (2013).

[20] Lv, X., Zhang, G. \& Fu, W. Highly Efficient Hydrogen Evolution Using $\mathrm{TiO}_{2} /$ Graphene Composite Photocatalysts. Protein Eng. 27:570-576 (2012).

[21] Vaclav S, Daniela P. $\mathrm{TiO}_{2}$-Graphene Nanocomposite as High Performace Photocatalysts. The Journal of Physical Chemistry C.115:25209-25218 (2011). 
[22] Huang Qingwu. Enhanced Photocatalytic Activity of Chemically Bonded $\mathrm{TiO}_{2} /$ Graphene Composites Based on the Effective Interfacial Charge Transfer through the $\mathrm{C}-\mathrm{Ti}$ Bond. ACS Catalysis.3:1477-1485 (2013).

[23] Wang, W. et al. A study on upconversion UV-vis-NIR responsive photocatalytic activity and mechanisms of hexagonal phase $\mathrm{NaYF}_{4}: \mathrm{Yb}^{3+}, \quad \mathrm{Tm}^{3+} @ \mathrm{TiO} 2$ core-shell structured photocatalyst. Appl. Catal., B. 144, 379-385 (2014).

[24] Wei Su, Xining Lu. Catalytic Reduction of NOX Over $\mathrm{TiO}_{2-}$ Graphene Oxide Supported with MnOX at Low Temperature. Catalysis Letters. 145:1466-1456 (2015).

[25] Ying Xu, Yanping Mo. The synergistic effect of graphitic N and pyrrolic $\mathrm{N}$ for the enhanced photocatalytic performance of nitrogen-doped graphene $/ \mathrm{TiO}_{2}$ nanocomposites. Applied Catalysis B: Environmental. 181:810-817 (2016).

[26] Wang, Y. et al. Low-temperature solvothermal synthesis of grapheme- $\mathrm{TiO}_{2}$ nanocomposite and its photocatalytic activity for dye degradation. Mater Lett. 134, 115-118 (2014).
[27] Zhaohui Wang. Probing paramagnetic species in titania-based heterogeneous photocatalysis by electron spin resonance (ESR) spectroscopy-A mini review. Chemical Engineering Journal.170:353-362 (2011).

[28] Panpranot J. Synthesis, Characterization, and Catalytic Properties of $\mathrm{Pd}$ and $\mathrm{Pd}-\mathrm{Ag}$ Catalysts Supported on Nanocrystalline $\mathrm{TiO}_{2}$ Prepared by the Solvothermal Method. Catalysis Letters.103:53-58 (2005).

[29] HeJian Leng. Investigation of the kinetics of a $\mathrm{TiO}_{2}$ photoelectrocatalytic reaction involving charge transfer and recombination through surface states by electrochemical impedance spectroscopy. The journal of physical chemistry. B.109:15008-15023 (2005).

[30] Lufeng Lu. Visible-Light-Driven Photodegradation of Rhodamine $\mathrm{B}$ on Ag-Modified BiOBr. Catalysis Letters.142:771-778 (2012). 